\title{
Neural Network Approach to Water-Stressed Crops Detection Using Multispectral WorldView-2 Satellite Imagery
}

\author{
Dubravko Ćulibrk, Predrag Lugonja, Vladan Minić, and Vladimir Crnojević \\ Faculty of Technical Sciences, University of Novi Sad, \\ Trg Dositeja Obradovica 6, 21000 Novi Sad, Serbia \\ \{dculibrk, lugonja, minic, crnojevic\}@uns.ac.rs \\ http://www.biosense.uns.ac.rs/
}

\begin{abstract}
The paper presents a method for automatic detection and monitoring of small waterlogged areas in farmland, using multispectral satellite images and neural network classifiers. In the waterlogged areas, excess water significantly damages or completely destroys the plants, thus reducing the average crop yield. Automatic detection of (waterlogged) crops damaged by rising underground water is an important tool for government agencies dealing with yield assessment and disaster control.

The paper describes the application of two different neural network algorithms to the problem of identifying crops that have been affected by rising underground water levels in WorldView-2 satellite imagery. A satellite image of central European region (North Serbia), taken in May 2010, with spatial resolution of $0.5 \mathrm{~m}$ and 8 spectral bands was used to train the classifiers and test their performance when it comes to identifying the water-stressed crops. WorldView- 2 provides 4 new bands potentially useful in agricultural applications: coastal-blue, red-edge, yellow and near-infrared 2. The results presented show that a Multilayer Perceptron is able to identify the damaged crops with $99.4 \%$ accuracy. Surpassing previously published methods.
\end{abstract}

Keywords: Water stress, Agriculture, Satellite imagery, Neural networks, Waterlogged farmland, Remote sensing.

\section{Introduction}

The advances in the satellite imaging technology provide researchers and practitioners with ever more data that needs to be processed to extract meaningful and useful information.

In agriculture, the applications of satellite imagery typically deal with crop monitoring [23] [5] 19] 15] 2]. A general goal is to be able to predict the yield of specific crops and monitor their health. Such information can be used directly by government agencies to ensure that the subsidies given to farmers are allocated correctly and that they are compensated for damage occurring in their fields. 
While satellite imagery for crop monitoring is a practice with more than 30 years of tradition [5] [19], the sensors have only recently gained resolution that allows for precision monitoring in the case of agriculture practices based on small land parcels [15] 2] 86] 23].

In the work presented here we address the problem of detecting small wetland areas in the farmland. Particularly, we are interested to detect these areas in the plains of Northern Serbia (Central Europe), where the small areas of wetland appear in the arable land as the consequence of the rise of a groundwater. This phenomenon emerges usually after the period of heavy rains, affects small mutually isolated areas, and disappears in a couple of weeks. Although temporary, it influences crop yields significantly and therefore it is necessary to discover it in order to perform damage assessment. In addition, farmers in Serbia are entitled to compensation if more than $30 \%$ of their crop is affected. Since crops are still present in the wetland areas, their stress due to excess water can be used to detect the stretches of waterlogged area within arable land. In addition, the presence of the crops in the water occludes the water surface, rendering the methods which wold rely on reflection from water inapplicable.

High-resolution 8-band images, provided by the remote sensing equipment mounted on the recently launched WorldView-2 satellite are used to detect crops damaged by excess water. Besides the standard red, green, blue and near-infra red band, WorldView-2 it offers new bands: costal blue, yellow, red-edge and an additional near-infra red band 11 . The new channels carry significant information one one is concerned with watelogged areas detection [18. In addition, multispectral images are available in high spatial resolution of $50 \mathrm{~cm}$, making them suitable for applications in areas with small-land-parcel-based agriculture.

Human-annotated ground truth was used to train 2 types of neural network classifiers to distinguish between pixels pertinent to waterlogged land and other pixels: Multi Layer Perceptron (MLP) and Radial Basis Function (RBF) neural nets. The experimental results presented show that the MLP approach achieves superior performance both in terms of accuracy and detection speed, when compared to previously published results [18]. RBF however, proved unsuitable for the task at hand, since it achieves accuracy below the simplest (logistic regression) classifier.

The rest of the paper is organized as follows: Section 2 provides an overview of the relevant published work. Section 3 provides the details of our approach. Section 4 describes the experiments conducted and the results achieved. Finally, Section 5 provides our conclusions.

\section{Related Work}

The problem of waterlogged areas detection falls into the scope of land-cover classification. Lu and Weng [10] provide a comprehensive and fairly recent survey of the different approaches used to distinguish between different types of cover. Within their taxonomy the proposed approach is supervised, non-parametric, per-pixel, spectral and makes hard decisions. This means that we use ground 
truth data created by human experts to train the classifier, make no assumptions about the data itself, consider single pixels with no notion of spatial dependencies and derive unambiguous classification decisions.

As far as precision agriculture applications are concerned, using satellite imagery for crop identification and crop-covered area estimation is a practice with more than 30 years of tradition [5] 19. Initially low-resolution Landsat MSS and TM data was used 5 19, 21, 14. As new satellites became available SPOT imagery [13] [1], Indian Remote Sensing (IRS) satellite data 44 16] and moderateresolution Moderate Resolution Imaging Spectroradiometer (MODIS) [9] 20] data has been used. More recently several studies have looked at using high-resolution satellite imagery from QuickBird [15] 2], IKONOS [8] and SPOT 5 [23].

Using neural networks for classification of multispectral image pixels was considered as early as 1992 [7. At the time, the authors of the study were concerned with the feasibility of training the network classifying the pixels of a satellite image in reasonable time, using the hardware available. The considered a single hidden layer feed-forward neural network trained and proposed an adaptive back-propagation algorithm, which reduced the training time from 3 weeks to 3 days, for a network with 24, 24 and 5 nodes in the input, hidden and output layer. At the time, a single input neuron was used for each bit in the RGB pixel value of a Landsat image. The authors concluded that the neural network is able to generalize well across different images, both real and synthetic. Since this early work there have been numerous applications of neural networks to classification of remotely sensed data. Mas and Flores provide an overview [12].

A recent study by Marchisio et al. [11 evaluated the capability of new WorldView-2 data, when it comes to general land-cover classification. They considered the relative predictive value of the new spectral bands in the WorldWiew2 sensor. Four types of classifiers were employed: logistic regression, decision trees, random forest and neural network classifiers. The study showed that new bands lead to an improvement of 5-20\% in classification accuracy on 6 different scenes. In addition, the authors proposed an approach to evaluate the relative contribution of each spectral band to the learned models and, thus, provide a list of bands important for the identification of each specific land cover class. Although the neural network classifiers used by Marchisio et al. [1] have not been described in detail, they state that they used a topology with two hidden layers, containing $10-20$ neurons. The neural network used was most likely a Multilayer Perceptron trained by back propagation.

Our work builds up on the study of Petrovic et al. [18. The authors used WorldView-2 data to address the problem of water-logged agricultural land detection. They evaluated logistic regression, Support Vector Machines (SVMs) and Genetic Programming (GP) classifiers. SVMs and GP achieved best classification results when cross validated (98,80\% and $98,87 \%$ accurate respectively). The GP solution was deemed the best by the authors, since it was able to achieve best classification and do it significantly faster than the SVM classifier that contained 6000 support vectors. Neural network classifiers were not considered in 
that study, and to the best of our knowledge were never applied to the problem of waterlogged area detection nor the problem of plant water-stress detection.

\section{Detecting Waterlogged Crops Using WorldView-2 Data}

\subsection{WorldView-2 Data}

The data used in this work is provided by commercial satellite WorldView-2. The image was made during July 2010 and covers the area of arable land from in the northern part of Serbia. Figure 1 shows visible light components of a part of the scene. The discolored irregular shaped blotches within the arable land correspond to waterlogged parts.

WorldView-2 is the first commercial satellite which provides eight highresolution spectral bands. Its sensors are sensitive to visible and near-infrared spectral bands and producce images of $50 \mathrm{~cm}$ resolution. This satellite detects radiation in following spectral bands: red $(630-690 \mathrm{~nm})$, green $(510-580 \mathrm{~nm})$, blue (450-510 nm), near-infrared 1 (NIR1 - 770-895 nm), coastal blue (400-450 nm), yellow (585-625 nm), red-edge (705-745 nm) and near-infrared 2 (NIR2 - 860$1040 \mathrm{~nm}$ ). The last four components provide additional value when compared to the data available from other commercial satellites and each of them has been designed with specific applications in mind. The costal blue is least absorbed by water, but it is absorbed by chlorophyll in healthy plants. NIR2 component to some extent resembles the characteristics of NIR1 component, initially aimed at vegetation detection and classification tasks, but is less influenced by atmospheric interference. Red-edge is especially designed for maximal reflection from vegetation and is intended for the measurement of plant health and classification of vegetation [3].

The data set used for training and testing of classifiers was derived based on ground truth, manually annotated on a part of a single satellite image covering an area of $10 \mathrm{~km}^{2}$. It contains environ 200,000 data samples, corresponding to the values of 8 bands for both waterlogged and non-waterlogged pixels, selected out some 25,000,000 pixels in the single scene. The values for each of the 8 bands are 16 bits wide. All values were normalized in our experiments.

\subsection{Neural Network Classification}

Two types of feed forward neural networks were considered for the classification of multispectral pixel values: Multilayer Perceptron (MLP) 6] and Radial Basis Function (RBF) [17.

Both architectures represent feed-forward networks the signals are propagated from the input to output neurons, with no backward connections. The MLP contained a single hidden layer and was trained using a back-propagation algorithms as detailed in 22 .

The parameters of the RBF network used were determined using k-means clustering. Both MLP and RBF implementation is available within the opensource data mining and artificial intelligence suite Wakaito Environment for 


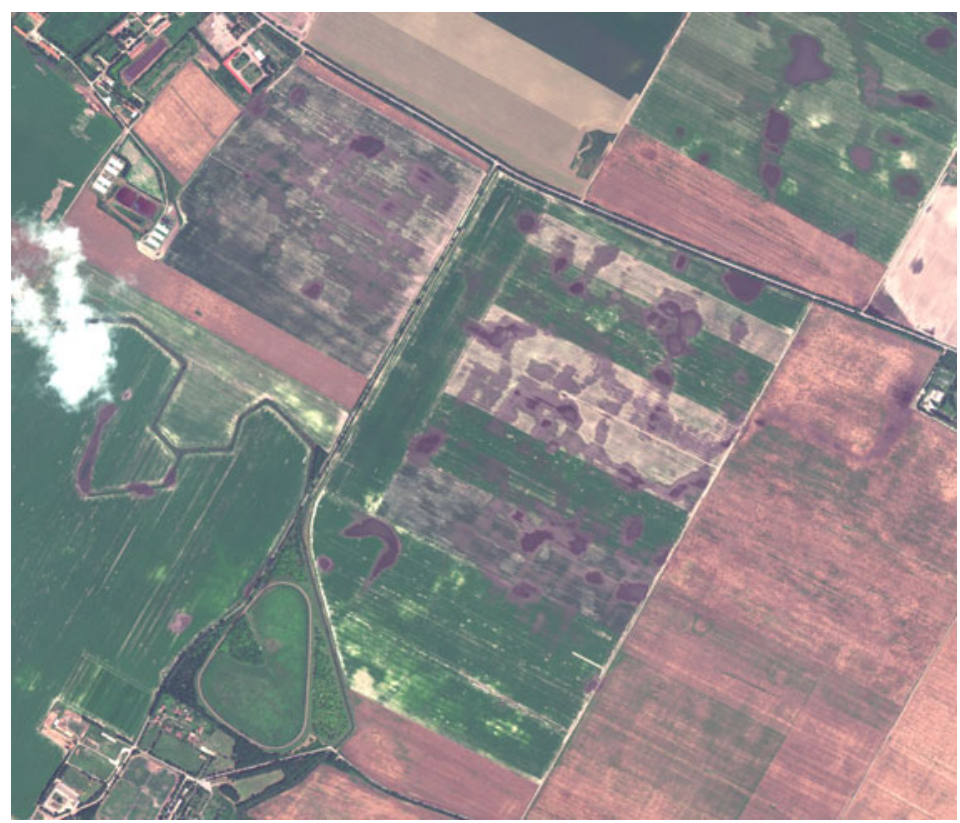

Fig. 1. Part of the satellite image used to derive the training data set and test the classifiers. Only red, green and blue bands are shown.

Knowledge Discovery (WEKA) 22]. This is the suite that has been used to conduct experiments detailed in Section 4 .

Once the neural networks are trained they are used to classify each pixel in the image separately.

\section{Experiments and Results}

To evaluate the performance of the two neural network models 10 -fold cross validation was used. The process involves holding out 10 percent of data in the training data set and using the rest to train the classifier. The $10 \%$ is used to test the model. The process is repeated 10 times and average performance measures are reported for each type of classifier.

The MLP contained 100 neurons in a single hidden layer, learning rate was set to 0.3 , momentum to 0.2 and 500 training epochs were used. The time taken to build the model on an Intel Core2Duo processor running at $2.93 \mathrm{GHz}$ was 6251 seconds. Table 1 provides the performance statistics for the MLP model: true-positive (TP) rate, false-positive (FP) rate, precision, recall, f-measure and Response Operating Characteristic (ROC) Area. The accuracy achieved by the MLP is $99.4043 \%$.

The RBF model was built much faster. It took only 43.69 seconds to train the network. Minimum standard deviation for the clusters was set to 0.1 , while the 
Table 1. Classification results for Multilayer Perceptron

\begin{tabular}{lcccccr}
\hline \multicolumn{7}{c}{ TP Rate FP Rate Precision Recall F-Measure ROC Area } \\
\hline Not waterlogged & 0.998 & 0.037 & 0.995 & 0.998 & 0.997 & 0.995 \\
Waterlogged & 0.963 & 0.002 & 0.987 & 0.963 & 0.975 & 0.995 \\
Weighted Avg. & 0.994 & 0.033 & 0.994 & 0.994 & 0.994 & 0.995 \\
\hline
\end{tabular}

Table 2. Classification results for RBF

\begin{tabular}{lcccccr}
\hline \multicolumn{7}{c}{ TP Rate FP Rate Precision Recall F-Measure ROC Area } \\
\hline Not waterlogged & 0.992 & 0.410 & 0.947 & 0.992 & 0.969 & 0.925 \\
Waterlogged & 0.590 & 0.008 & 0.904 & 0.590 & 0.714 & 0.925 \\
Weighted Avg. & 0.944 & 0.362 & 0.942 & 0.944 & 0.938 & 0.925 \\
\hline
\end{tabular}

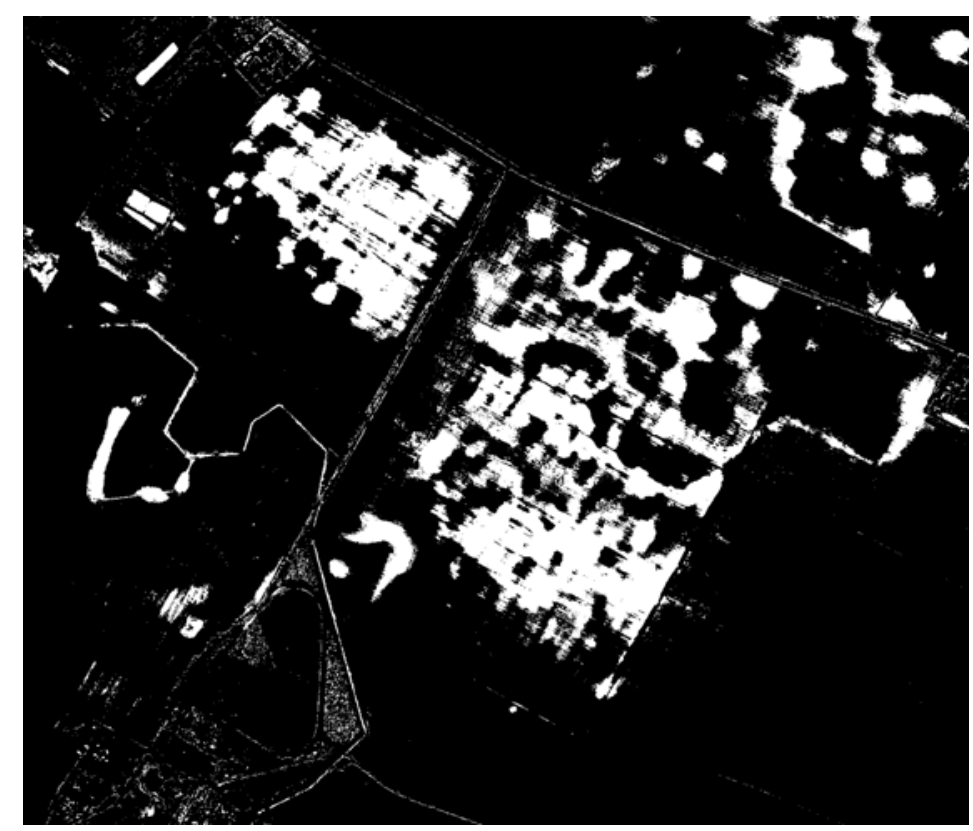

Fig. 2. MLP classification result for the image shown in Fig. 1

ridge parameter for the logistic regression was set to $10^{-} 8$. Table 1 provides the performance statistics for the RBF model. The accuracy achieved by the RBF is significantly lover than that of the MLP (94.3538\%).

Figures 2 and 3 show the classification results for a part of the satellite image shown in Figure 1. The parts of the scene classified as waterlogged are indicated by white pixels. Since the waterlogged areas represent a relatively small part of the overall area, the classification result shown in two images differs significantly, although the difference in accuracy between two methods is just $5 \%$. 


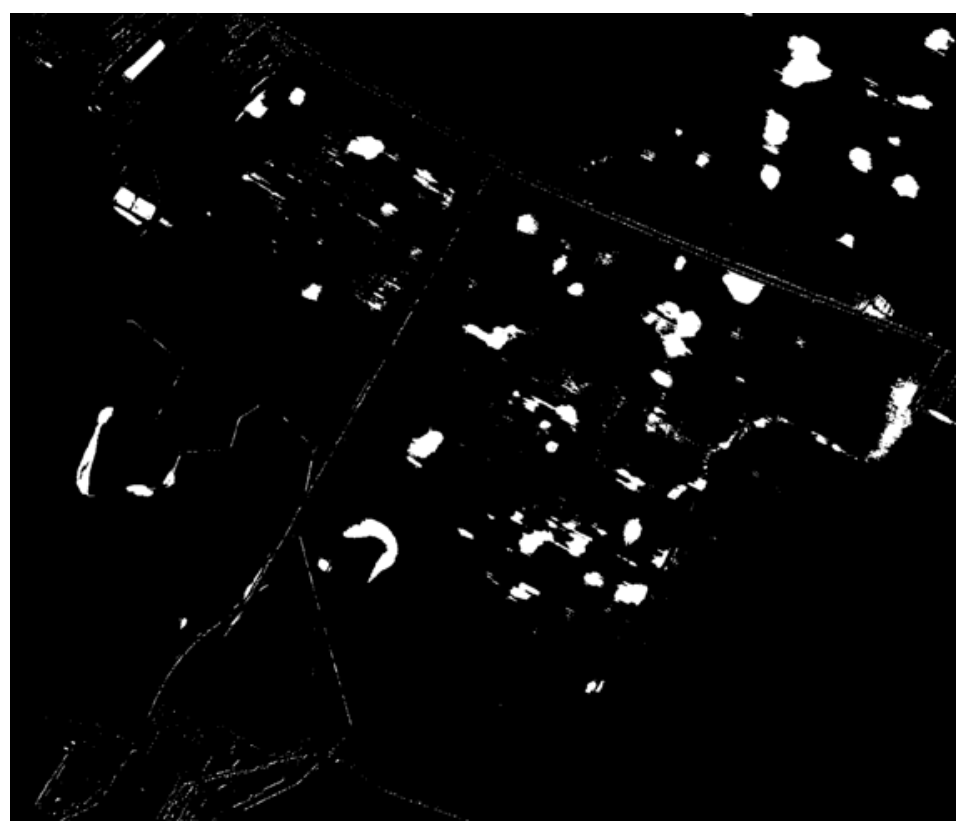

Fig. 3. MLP classification result for the image shown in Fig. 1

When compared to the results of other classifiers as reported in [18, the MLP achieved results superior to any of the classifiers reported there. The best classifier reported in the previous study was achieved by Genetic Programming and its accuracy was $98.87 \%$ on the same data set. Unfortunately the RBF classifier performed worse even than the simple logistic regression, which achieved $97.58 \%$ accuracy, as reported previously. This can only be attributed to the errors in the $\mathrm{k}$-means clustering used to position the kernels of the RBF.

\section{Conclusion}

The application of two different neural network classifiers to the problem of detecting waterlogged farmland using multispectral satellite imagery, has been evaluated in the study presented.

Using imagery acquired by the new WorldView-2 satellite, we showed that Multilayer Perceptron can achieve accuracy superior to that of other published approaches and classify $99.4 \%$ of the pixels in the scene accurately.

RBFs have not proved successful when applied to the problem at hand, since the k-means procedure used to position the kernel functions was unable to cope. Other ways of doing this, such as random sampling from the training data or orthogonal least square learning should be considered if RBFs are to be used in this scenario. 
As a direction for further research, the methodology could be evaluated in terms of detecting less pronounced effects of water stress, where plants are not irreparably damaged. It could also be possible to design crop yield estimation models, akin to the methodology presented in [15].

\section{References}

1. Büttner, G., Csillag, F.: Comparative study of crop and soil mapping using multitemporal and multispectral SPOT and Landsat Thematic Mapper data. Remote Sensing of Environment 29(3), 241-249 (1989)

2. Castillejo-González, I., López-Granados, F., García-Ferrer, A., Peña-Barragán, J., Jurado-Expósito, M., de la Orden, M., González-Audicana, M.: Object-and pixelbased analysis for mapping crops and their agro-environmental associated measures using QuickBird imagery. Computers and Electronics in Agriculture 68(2), 207-215 (2009)

3. DigitalGlobe: The Benefits of the 8 Spectral Bands of WorldView-2. whitepaper (2009)

4. Dutta, S., Sharma, S., Khera, A., et al.: Accuracy assessment in cotton acreage estimation using Indian remote sensing satellite data. ISPRS Journal of Photogrammetry and Remote Sensing 49(6), 21-26 (1994)

5. Hanuschak, G.: Obtaining timely crop area estimates using ground-gathered and LANDSAT data. Dept. of Agriculture, Economics, Statistics, and Cooperatives Service: for sale by the Supt. of Docs., US Govt. Print. Off. (1979)

6. Haykin, S.: Neural Networks: A Comprehensive Foundation. Macmillan, New York (1994)

7. Heermann, P., Khazenie, N.: Classification of multispectral remote sensing data using a back-propagation neural network. IEEE Transactions on Geoscience and Remote Sensing 30(1), 81-88 (1992)

8. Helmholz, P., Rottensteiner, F., Heipke, C.: Automatic qualtiy control of cropland and grasland GIS objects using IKONOS Satellite Imagery. IntArchPhRS (38) Part 7, 275-280 (2010)

9. Lobell, D., Asner, G.: Cropland distributions from temporal unmixing of MODIS data. Remote Sensing of Environment 93(3), 412-422 (2004)

10. Lu, D., Weng, Q.: A survey of image classification methods and techniques for improving classification performance. International Journal of Remote Sensing 28(5), 823-870 (2007)

11. Marchisio, G., Pacifici, F., Padwick, C.: On the relative predictive value of the new spectral bands in the WorldWiew-2 sensor. In: 2010 IEEE International Geoscience and Remote Sensing Symposium (IGARSS), pp. 2723-2726. IEEE, Los Alamitos (2010)

12. Mas, J., Flores, J.: The application of artificial neural networks to the analysis of remotely sensed data. International Journal of Remote Sensing 29(3), 617-663 (2008)

13. Murakami, T., Ogawa, S., Ishitsuka, N., Kumagai, K., Saito, G.: Crop discrimination with multitemporal SPOT/HRV data in the Saga Plains, Japan. International Journal of Remote Sensing 22(7), 1335-1348 (2001)

14. Oetter, D., Cohen, W., Berterretche, M., Maiersperger, T., Kennedy, R.: Land cover mapping in an agricultural setting using multiseasonal Thematic Mapper data. Remote Sensing of Environment 76(2), 139-155 (2001) 
15. Pan, G., Sun, G., Li, F.: Using QuickBird imagery and a production efficiency model to improve crop yield estimation in the semi-arid hilly Loess Plateau, China. Environmental Modelling \& Software 24(4), 510-516 (2009)

16. Panigrahy, S., Sharma, S.: Mapping of crop rotation using multidate Indian Remote Sensing Satellite digital data. ISPRS Journal of Photogrammetry and Remote Sensing 52(2), 85-91 (1997)

17. Park, J., Sandberg, I.: Universal approximation using radial-basis-function networks. Neural Computation 3(2), 246-257 (1991)

18. Petrovic, N., Lugonja, P., Culibrk, D., Crnojevic, V.: Detection of Wet Areas in Multispectral Images of Farmland. In: The Seventh Conference on Image Information Mining: Geospatial Intelligence from Earth Observation, pp. 21-24 (April 2011)

19. Ryerson, R., Dobbins, R., Thibault, C.: Timely crop area estimates from Landsat. Photogrammetric Engineering and Remote Sensing 51(11), 1735-1743 (1985)

20. Wardlow, B., Egbert, S.: Large-area crop mapping using time-series MODIS 250 $m$ NDVI data: An assessment for the US Central Great Plains. Remote Sensing of Environment 112(3), 1096-1116 (2008)

21. Williams, V., Philipson, W., Philpot, W.: Identifying vegetable crops with Landsat Thematic Mapper data. Photogrammetric Engineering and Remote Sensing 53, 187-191 (1987)

22. Witten, I.H., Frank, E.: Data Mining: Practical machine learning tools and techniques, 2nd edn. Morgan Kaufmann, San Francisco (2005)

23. Yang, C., Everitt, J., Murden, D.: Evaluating high resolution SPOT 5 satellite imagery for crop identification. Computers and Electronics in Agriculture (2011) 